\title{
ALIEN SYMBOLS FOR ALIEN LANGUAGE: ITERATED LEARNING IN A UNIQUE, NOVEL SIGNAL SPACE
}

\author{
Christine CUSKLEY ${ }^{* 1}$ \\ *Corresponding Author: ccuskley@gmail.com \\ ${ }^{1}$ Centre for Language Evolution, The University of Edinburgh, UK
}

Studies in iterated learning of artificial 'alien' languages show that structure in language can arise from the process of cultural transmission (Kirby, Cornish, \& Smith, 2008). Structure can accumulate not only in iterated artificial language learning (ALL) tasks, but also in iterated sequence learning tasks which are not explicitly linguistic (e.g., they contain no meanings, and do not use linguistic stimuli). Meaningless sequences of colours become more structured and more learnable over time (Cornish, Smith, \& Kirby, 2013), reproduction of rhythmic sequences results in increased structure, learnability, and emergence of 'musical universals' (Ravignani, Delgado, \& Kirby, 2016), and transmission of slide whistles can result increased structure (Verhoef, Kirby, \& Boer, 2014). Where signals are mapped onto meanings, the relationships between these two spaces can have significant effects on the resulting structure (Little, Erylmaz, \& Boer, 2017). While the signals and modalities with which iterated learning studies are conducted have expanded considerably in recent years, how properties of signal spaces interact with biases of learners in the emergence of structure is relatively under-studied.

To further investigate the effect of signal space on the evolution of structure and learnability, we used a completely novel set of graphical symbols called Ferros: a set of 137 abstract graphemes created using ferrofluid ink and visually unlike e.g., Roman orthography: move in a virtual two dimensional palette which produces a different symbol depending on their location within a square. The space is structured along two axes corresponding to the number of contours and nodes in each Ferro. Much like the phonetic space of consonants, distance between Ferros encodes similarity, but the forms are discrete. Ferros are signals which are entirely foreign to participants, who have to learn not only to use the apparatus which produces Ferros, but also what the relevant features of Ferros are.

The Ferro palette was used in an iterated sequence copying task involving hundreds of participants: each participant had three minutes to copy as many 3character Ferro sequences as they could. Each sequence faded slowly after presen- 
tation, providing an additional pressure for quick reproduction. Participants were confined to producing three characters (i.e., sequences could not vary in length), and were provided with feedback on accuracy at each trial.

The initial sequences were pseudo-random, consisting of graphemes with either large articulation spaces in the palette or small, specific articulation spaces. Overall, 672 sequences were produced across 76 chains, each chain being between 5 and 10 generations. Reproduction error was measured as mean Euclidean distance in the palette from the target sequence to the produced sequence, and examined using a linear mixed effects model with generation and articulation space size as fixed effects.

Inclusion of generation and articulation space size improved the model fit significantly over a null model $\left(\chi^{2}=4.53, p=0.03\right)$. Error was higher overall in chains which started with small articulation spaces $(\beta=0.1, \mathrm{SE}=0.04, t=2.5)$, indicating that participants found these harder to reproduce. Error decreased over generations in the small articulation space condition $(\beta=-0.018, \mathrm{SE}=0.006$, $t=-3.035)$, but there was no such effect of generation for sequences which started with large articulation spaces.

Error decreased despite an overall retention of high sequence entropy: in other words, most sequences retained three different characters throughout a chain. The size of the area of the articulation space of a particular Ferro grapheme was significantly correlated with copying accuracy, and sequences in small articulation chains moved towards the larger articulation spaces over time, suggesting that larger articulation spaces make for greater ease of articulation.

The current study shows transmission can lead to increased learnability even in a completely novel signal system that participants have to learn from scratch in the process of transmission. In this case, the biases which drive increased learnability seem to derive primarily from the shape of the signal space: articulation area of a particular Ferro correlates significantly with its learnability (operationalised as reproduction error), and sequences gravitated towards larger articulation spaces over 'time'. The shape of the space was perhaps especially influential in the current task because participants had no prior experience with Ferros, and so had fewer (or less influential) prior biases relative to other iterated learning tasks, particularly those that utilise non-words and leverage existing linguistic knowledge. This has potential implications for the co-evolution of language and speech: constraints of the signal space may be more influential in early stages of emergence as 'speakers' are not only reproducing utterances, but also learning how to manipulate a novel signal space. This study shows that the Ferro palette has broad applications in language evolution research as a system of truly 'alien' symbols to study 'alien' language learning, emergence, and evolution. 


\section{Acknowledgements}

This research was supported by British Academy Postdoctoral Fellowship grant no. pf150065. The author thanks Sean Roberts, Stefan Hartman, Michael Pleyer, and Hannah Little for valuable input with on the interface design, implementation, and assistance with translation.

\section{References}

Cornish, H., Smith, K., \& Kirby, S. (2013). Systems from sequences: an iterated learning account of the emergence of systematic structure in a nonlinguistic task. In M. Knauff, M. Pauen, N. Sebanz, \& I. Wachsmuth (Eds.), Proceedings of the 35th annual conference of the cognitive science society (p. 340-345). Austin, TX: Cognitive Science Society.

Kirby, S., Cornish, H., \& Smith, K. (2008). Cumulative cultural evolution in the laboratory: An experimental approach to the origins of structure in human language. Proceedings of the National Academy of Sciences, 105(31), 10681-10686.

Little, H., Erylmaz, K., \& Boer, B. de. (2017). Signal dimensionality and the emergence of combinatorial structure. Cognition, 168, 1 - 15.

Ravignani, A., Delgado, T., \& Kirby, S. (2016). Musical evolution in the lab exhibits rhythmic universals. Nature Human Behvaiour, 1.

Verhoef, T., Kirby, S., \& Boer, B. de. (2014). Emergence of combinatorial structure and economy through iterated learning with continuous acoustic signals. Journal of Phonetics, 43, 57 - 68. 